International Journal of Engineering, Science and Technology

Vol. 9, No. 2, 2017, pp. 15-25
INTERNATIONAL

JOURNAL OF

ENGINEERING,

SCIENCE AND

TECHNOLOGY

www.ijest-ng.com

www.ajol.info/index.php/ijest

(C) 2017 MultiCraft Limited. All rights reserved

\title{
Optimized tuning of contention window for IEEE 802.11 WLAN
}

\author{
B. Nithya*, A. Justin Gopinath, Venkatesh Kameswaran, P. Yogesh \\ Department of Computer Science and Engineering, National Institute of Technology, Tiruchirappalli, INDIA \\ *Corresponding Author: e-mail:nithya@nitt.edu,Tel 0431-2503214
}

\begin{abstract}
In wireless networks, the size of Contention Window $(\mathrm{CW})$ plays a vital role as it changes the throughput and delay of the networks considerably. The proposed Collision Ratio based BackOff (CRBO) algorithm uses collision ratio to suggest CW updation after the successful and unsuccessful transmission events. This leads to proper scheduling of transmission when more than one node attempt to capture the shared medium. The strength of the proposed algorithm is analyzed using NS2 simulator in terms of throughput, delay, packet loss and fairness. The simulation results prove that the proposed algorithm outperforms the existing algorithms under heavy and low traffic in random and grid topology.
\end{abstract}

Keywords: Wireless networks, contention window, collision rate, backoff, NS2.

DOI: http://dx.doi.org/10.4314/ijest.v9i2.2

\section{Introduction}

One of the most frequently used standards for Wireless networks is the IEEE 802.11 standard (Brenner, 1997). In a shared medium, in which all stations compete for access, and only one of the stations can transmit at any given instant of time. Moreover, if two or more stations attempt to send data packets at the same time, collision will happen and the packet is lost. In case of wireless networks, it is difficult to distinguish between collision of packets and external interference which leads to detecting collision highly complicated (Thorpe et al, 2014). But, the 802.11 standard defines the access mechanism of Carrier Sense Multiple Access with Collision Avoidance (CSMA/CA) and offers the Distributed Coordination Function (DCF). In DCF (Jochen, 2003), one of the most fundamental methods, all stations compete for the transmission medium. If a station finds the busy medium, back-off algorithm is executed to minimize collisions.

The DCF mechanism is as follows: If a station is about to send a packet, the smallest CW is taken and in case of a collision of the packet, the station chooses an exponentially increased CW. Such cases occur when there is heavy traffic i.e many stations access the shared wireless medium at the same physical location and time (Zhai et al, 2006). Once a station transmits its packet successfully, its back-off window is reset to the smallest size for future transmissions; but, this fares poorly in case of heavy traffic (Natkaniec et al, 2013). This paper tweaks this algorithm to some extent by changing the deciding factors and size of the contention window.

\section{Literature Review}

As mentioned in the previous section, the limitations of BEB are reduced by many enhanced algorithms proposed in the literature. Some of them along with demerits are discussed in the following paragraphs. Modified Algorithm for DCF (MADCF) under heavy traffic is proposed in (Elarbaoui et al, 2008). In this algorithm, the number of retransmissions is taken into account while calculating the contention window size after a successful transmission. If the number of retransmissions is greater than $\mathrm{n}$, the contention window size is reset to Wn after a successful transmission else reverts to zero. Bharghavan et al. proposed a new algorithm in MACAW scheme termed as Multiple Increase and Linear Decrease (MILD) backoff algorithm (Bharghavan et al, 
1994). In MILD, the CW size is multiplied with constant factor 1.5 instead of doubling it, when there is an unsuccessful transmission. After the successful transmission, CW size is decreased linearly by 1 instead of reset to 0 . By this method, the collision probability is reduced and simulation result (Bharghavan et al, 1994) shows that MILD is better than the BEB algorithm over large network.

Deng et al. (2004) proposed an algorithm called Linear Increase and Linear Decrease (LILD) (Deng et al., 2004) which is an extension of MILD. This protocol follows the same principle as MILD when successful transmission, where as in case of unsuccessful transmission the algorithm updates $\mathrm{CW}$ by $\mathrm{CW}+\mathrm{CWmin}$ instead of multiplying by 1.5. It avoids the problem of slow linear change over failed transmission and provides good throughput for large network.

Deng et al. (2004) extended their work with new approach called Linear/Multiplicative Increase and Linear Decrease (LMILD) (Huang et al., 2012) in which the failed nodes increases their CW multiplicatively while other neighbor nodes increases CW linearly. There is no packet reception during collision since each node uses an additional information which generates carrier sensing signal. In their study, LMILD outperforms BEB and MILD schemes in wide range of active nodes in networks. However, the above all algorithms focused on saturated traffic loads of wireless networks. Alkadeki et al. (2016) worked on non-saturated traffic loads and proposed dynamic control backoff time algorithm, implemented using Equilibrium Point Analysis (EPA) model. In DCBTA, low or heavy traffic load is determined using CWthreshold value. Upon unsuccessful transmission, if the traffic load is low, then CW size is doubled as BEB algorithm whereas if traffic load is high, the CW size is incremented by $2 * \mathrm{CW}+2$ which decreases collision probability, thus increasing throughput. Huang et al. (2016) addressed poor network fairness of BEB and proposed new algorithm termed as synchronized contention window ( $\mathrm{SCW}$ ) -based backoff algorithm. When the channel state is changed, the contending stations are synchronized by resetting the $\mathrm{CW}$. Hence, it guarantees that each station gets equal chance for channel access in next contention. Zhang et al. (2016) proposed a backoff algorithm based on self-adaptive contention window update factor. The algorithm modifies the updating factor of $\mathrm{CW}$ based on the current network load. They developed theoretical model using two dimensional Markov chain on backoff procedure.

The algorithm proposed by Kunho et al. (2012) measures optimized contention window in both saturated and unsaturated conditions. In saturated condition, $\mathrm{CW}$ size is estimated using channel busyness ratio to maximize the network throughput. Whereas in unsaturated condition, based on status of the current slot (idle or busy), two CW (WI and WB) values are determined to minimize the delay. Additionally, the protocol introduces the priorities in accessing the channel according to traffic classes. Bononi et al. (2004) proposed asymptotically optimal backoff that updates contention window size with respect to the current contention level by estimating the slot utilization and average size of transmitted frames. Measuring number of active stations accurately is difficult and hence the algorithm uses sampled p-persistent backoff interval from a geometric distribution with probability p. Each station updates the slot utilization in every backoff interval and uses an additional control parameter called probability of transmission before any transmission. The comparison of above mentioned algorithms is summarized in Table 1. It is inferred from the aforementioned discussion that updating $\mathrm{CW}$ value plays a predominant role in wireless network performance. By considering all these facts and drawbacks of existing algorithms as mentioned in Table 1, an adaptive collision Ratio based BackOff (CRBO) algorithm is proposed to enhance throughput and fairness by minimizing delay and packet loss.

\section{Proposed Algorithm}

The proposed CRBO algorithm intents to afford an efficient medium access control by proper scheduling of transmissions among competing stations. This algorithm uses collision ratio as a metric to indicate the network contention level in order to tune the pace of CW adjustments upon collisions and successful transmissions under heavy traffic network condition. To achieve this, every node keeps track of number of successful transmissions $\left(\operatorname{Tran}_{\mathrm{Suc}}\right)$ and collided $\operatorname{transmissions}\left(\operatorname{Tran}_{\mathrm{Col}}\right)$. These two local information are readily available and also reliable. Each node can have these parameters from its own experience without using complex calculation and run time measurements. As a consequent, protocol overhead, processing time and energy consumption are minimized. With the aid of above mentioned static information, collision ratio as in (1) is calculated to determine the next $\mathrm{CW}$ size.

$$
\text { Collision ratio }=\frac{\operatorname{Tran}_{\mathrm{Col}}}{\operatorname{Tran}_{\mathrm{Col}}+\operatorname{Tran}_{\text {Suc }}}
$$

This parameter correctly predicts the current network condition in heavily congested network. The higher collision ratio reveals high degree of contention and congestion in the network. In this situation, the difference between two consecutive CW sizes must be large enough to avoid further collisions. This will be achieved by faster $\mathrm{CW}$ increments. In other case, the lower collision ratio depicts less contention degree which requires adequate $\mathrm{CW}$ increments to avoid end to end delay. With these considerations, the proposed CW adjustments using eq(1) upon collisions and successful transmission are elaborated in the following subsections.

3.1 CW adjustments upon collisions: When a node experiences collision, collision ratio is examined. If it is less than threshold value, contention and congestion level in the network is under the controllable margin. In this case, $\mathrm{CW}$ needs to be incremented slowly to minimize end-to-end delay. The linear increment by the factor of 32 is recommended by the proposed CRBO algorithm and backoff time is chosen from the newly estimated CW range as shown in (2). 


$$
\text { CWnew }=\min (\mathrm{CWprev}+32, \mathrm{Cwmax})
$$

If collision rate is greater than the threshold value, it gives the sign of overloaded network condition. In this scenario, the immediate retransmissions need to be avoided to minimize the collision probability. Similar to BEB, the proposed algorithm exponentially increases $\mathrm{CW}$ size as shown in (3) to have larger $\mathrm{CW}$ range. As a result, $\mathrm{CW}$ size chosen from this expanded range is high which leads to longer waiting time. Moreover, from this enlarged $\mathrm{CW}$ range, the selection of similar $\mathrm{CW}$ size by more than one node is also effectively controlled. Hence the collision among competing stations is minimized.

$$
\text { CWnew }=\min ((\mathrm{CWprev}<<1)+1, \mathrm{CWmax})
$$

\begin{tabular}{|c|c|c|c|c|c|c|}
\hline \multicolumn{2}{|c|}{$\begin{array}{l}\text { Algorithm } \\
\text { Name }\end{array}$} & $\begin{array}{c}\text { CW increment upon } \\
\text { collision }\end{array}$ & $\begin{array}{l}\text { CW decrement upon } \\
\text { success }\end{array}$ & $\begin{array}{c}\text { No. of } \\
\text { nodes \& } \\
\text { topology }\end{array}$ & $\begin{array}{c}\text { Achieved } \\
\text { enhancements }\end{array}$ & $\begin{array}{l}\text { Problem/ } \\
\text { Drawbacks }\end{array}$ \\
\hline \multicolumn{2}{|c|}{ MILD } & $\mathrm{CW}=\min \left(\mathrm{CW}^{*} 2, \mathrm{CW} \max \right)$ & $\begin{array}{c}\mathrm{CW}=\max (\mathrm{CW}- \\
\mathrm{CWmin}, \mathrm{CWmin})\end{array}$ & $4-40$ & Better than BEB & $\begin{array}{c}\text { Suited for } \\
\text { saturated traffic }\end{array}$ \\
\hline \multicolumn{2}{|l|}{ LILD } & $\begin{array}{c}\mathrm{CW}=\min \\
(\mathrm{CW}+\mathrm{CW} \min , \mathrm{CW} \max )\end{array}$ & $\begin{array}{c}\mathrm{CW}=\max (\mathrm{CW}- \\
\mathrm{CWmin}, \mathrm{CWmin})\end{array}$ & $4-40$ & $\begin{array}{l}\text { good quality for } \\
\text { larger network }\end{array}$ & $\begin{array}{c}\text { Suited for } \\
\text { saturated traffic }\end{array}$ \\
\hline \multicolumn{2}{|c|}{ LMILD } & $\begin{array}{l}\mathrm{CW}=\min \left(\mathrm{mc}^{*} \mathrm{CW}, \mathrm{CW} \max \right) \\
\mathrm{CW}=\min (\mathrm{CW}+\mathrm{lc}, \mathrm{CW} \max )\end{array}$ & $\begin{array}{c}\mathrm{CW}=\max (\mathrm{CW}-\mathrm{ls}, \\
\mathrm{CWmin})\end{array}$ & 128 & $\begin{array}{l}\text { better than BEB for } \\
\text { large network ( } 87 \% \\
\text { throughput) }\end{array}$ & $\begin{array}{c}\text { Suited for } \\
\text { saturated traffic }\end{array}$ \\
\hline \multirow{2}{*}{$\begin{array}{l}\text { DCBT } \\
\text { A }\end{array}$} & $\begin{array}{l}\mathrm{L} \\
\mathrm{T}\end{array}$ & $\mathrm{CW}=\mathrm{CW} * 2$ & $\mathrm{CW}=\mathrm{CW}-1$ & $50-100$ & $\begin{array}{l}\text { Throughput increase } \\
\text { by } 10 \% \text { than BEB }\end{array}$ & \multirow{2}{*}{$\begin{array}{l}\text { Not considered } \\
\text { different } \\
\text { network } \\
\text { environment }\end{array}$} \\
\hline & $\begin{array}{l}\mathrm{H} \\
\mathrm{T}\end{array}$ & $\mathrm{CW}=\mathrm{CW} * 2+2$ & $\mathrm{CW}=\mathrm{CW}-2$ & $50-100$ & $\begin{array}{c}\text { Throughput increase } \\
\text { by } 3 \% \text { than BEB }\end{array}$ & \\
\hline \multicolumn{2}{|l|}{$\mathrm{SCW}$} & $\mathrm{CW}=\min (\mathrm{CW} \max , 2 * \mathrm{CW})$ & $\mathrm{CW}=\underset{\mathrm{CWax}(\mathrm{CW} \min )}{ }$ & $\begin{array}{c}4-40 \\
\text { Single- } \\
\text { hop } \\
\text { network }\end{array}$ & $\begin{array}{l}\text { Fairness index } \\
\text { nearly equal to } 1 \\
\text { and suitable for } \\
\text { dense stations }\end{array}$ & $\begin{array}{l}\text { For small } \\
\text { network the } \\
\text { delay is } \\
\text { increased. }\end{array}$ \\
\hline \multicolumn{2}{|c|}{ SACW } & $\mathrm{CW}=\min (\mathrm{c} . \mathrm{CW}$ old, $\mathrm{CW} \max )$ & $\begin{array}{c}\mathrm{CW}=\underset{\max (\mathrm{CWold} / \mathrm{c},}{\mathrm{CWmin})}\end{array}$ & $5-100$ & $\begin{array}{l}\text { Better channel } \\
\text { utilization }\end{array}$ & $\begin{array}{l}\text { Fairness not } \\
\text { addressed }\end{array}$ \\
\hline \multicolumn{2}{|c|}{ CCBCWA } & \multicolumn{2}{|c|}{ For Saturated, $\mathrm{Ws}=\left\{\begin{array}{l}\mathrm{W}_{0}, \quad \text { if } \sum_{i=1}^{k} u_{i}=0 \\
\mathrm{~W} \times \sum_{i=0}^{k} u_{i}, \quad \text { otherwise }\end{array}\right.$} & 50 & $\begin{array}{l}\text { Better delay and } \\
\text { throughput }\end{array}$ & $\begin{array}{l}\text { 1.Tested only } \\
\text { for small size } \\
\text { network } \\
\text { 2.Did not } \\
\text { differentiate } \\
\text { transmission } \\
\text { event }\end{array}$ \\
\hline \multicolumn{2}{|l|}{ AOB } & \multicolumn{2}{|c|}{$\begin{array}{l}\text { Station transmit with probability } \\
\qquad \mathrm{P}-\mathrm{T}(\mathrm{ACL}(\mathrm{q}), \mathrm{S}-\mathrm{U}, \mathrm{N}-\mathrm{A})=1-\min \left(1, \frac{S-U}{A C I(q)}\right)^{\mathrm{N}-\mathrm{A}} \\
\text { Otherwise, reschedule with probability 1- } \text { P_T }^{-\mathrm{T}}\end{array}$} & 200 & $\begin{array}{l}\text { Increased cannel } \\
\text { utilization }\end{array}$ & $\begin{array}{l}\text { 1.Probabilistic } \\
\text { way to tune } \mathrm{CW} \\
\text { size } \\
\text { 2.Fairness not } \\
\text { addressed }\end{array}$ \\
\hline
\end{tabular}

Table 1. Comparison of Backoff Algorithms

3.2 CW adjustments after successful transmissions: Under heavy traffic condition, it is highly mandatory to adjust $\mathrm{CW}$ after having successful transmissions. As in BEB, if CW reverts to its minimum $\mathrm{CW}$ (CWmin), the following problems are unavoidable. (i)The stations which had successful transmission always preempts longer waiting stations. This leads to channel capture phenomenon.

(ii) Newly entered stations and stations with successful transmissions may select the more or less same CW size from the limited $\mathrm{CW}$ range $(0, \mathrm{CWmin})$. As a consequent, the collisions among these stations are inevitable. 
(iii) The difference between CW size of a new packet and a packet which faces many collisions leads to severe delay jitter.

(iv) There is a high probability for service starvation in congested network.

To overcome above mentioned problems, several algorithms adopted different $\mathrm{CW}$ adjustments to be followed after successful transmission as discussed in Section 2. But the limitation in these algorithms is that the current network condition is not considered while estimating CW size. As depicted in Table 1, they decremented CW value either linearly or exponentially. To mitigate these difficulties, the proposed CRBO algorithm suggests $\mathrm{CW}$ updation based on collision rate.

As in BEB, CW resetting mechanism is utilized if the collision ratio is less than or equal to threshold value. The lesser collision ratio indicates the lesser number of active stations. In this case, the waiting time is chosen from the shorter range $(0, \mathrm{CWmin})$ to minimize the channel access delay which further leads to higher network throughput and channel utilization. Otherwise, $\mathrm{CW}$ size is estimated as shown in the proposed eq (4).

$$
\mathrm{CWnew}=\max \left(\mathrm{CWmin} *\left(2^{\wedge} \mathrm{rc}\right)+32, \mathrm{CWmin}\right)
$$

By using polynomial function of retransmission count (rc), the new CW size is determined. This forces the stations to wait for sufficient amount of time before immediately starting next transmissions. Meantime, the newly entered stations or stations with lesser waiting time may get the channel access. This leads to fairness among competing stations.

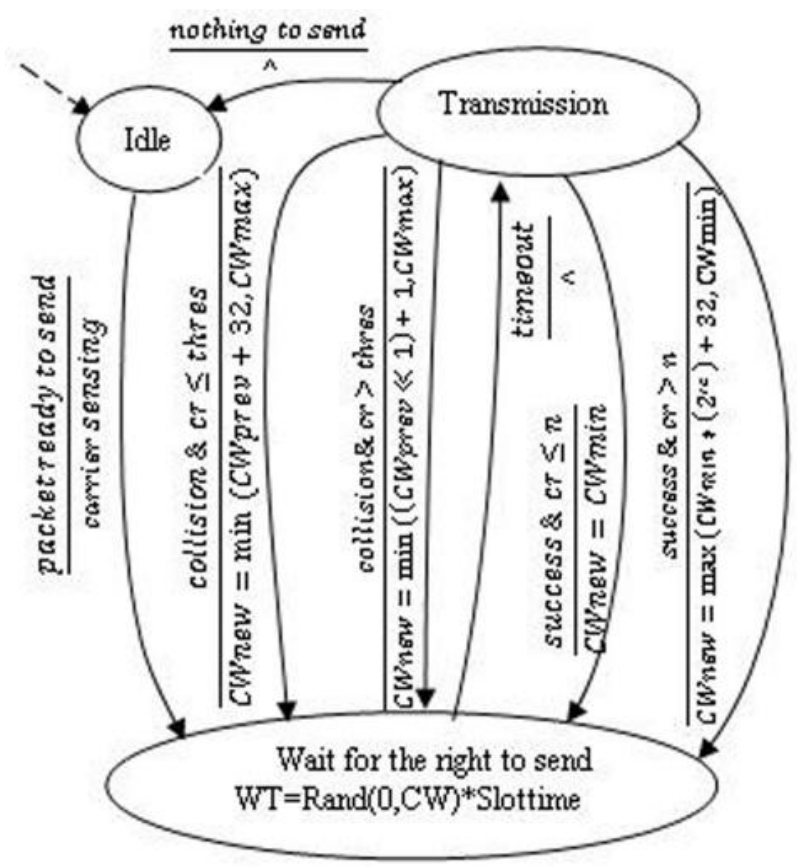

Figure 1. FSM diagram of Proposed Algorithm

The Finite State Machine (FSM) diagram for the proposed CRBO algorithm is shown in Figure 1. The event and the corresponding action are shown above and below the horizontal line respectively. If there is no corresponding event, then the symbol is used. Initially, node will be in the Idle state. If the node has packet to send, it goes to Wait for the right to send state. After the timeout event, it goes to Transmission state. Based on the status of transmission and collision ratio, CWnew value is chosen. The new waiting time (WT) is estimated according to this value as depicted in Figure 1. Once all the data have been transmitted, node goes to Idle state.

\section{Simulation and Results}

The focal points of this section are to assess the performance of the proposed CRBO algorithm through NS2 simulator (Teerawat et al, 2009) and compare the results with BEB and MADCF. The behavior of these three algorithms in terms of throughput, delay, and packet loss and fairness index are measured with varying number of nodes under random and grid topology. The scale of the random topology is varied from 80 nodes to 160 nodes with $10 \mathrm{~m} / \mathrm{s}$ as mobility speed. Since the nodes are freely moving, the random topology depicts the dynamic nature of wireless mobile network. Whereas in grid topology, a static wireless network is constructed with $49,64,81,100$ and 121 nodes. To retain the structure of the topology throughout the simulation, node mobility is not imposed into the grid topology. Figure 2 and 3 show the NS2 screenshot of random and grid topology of 100 nodes 
respectively. . In these screenshot, the circles are the transmission range and the arrowhead lines show the transmission directions of the corresponding node. In this simulation, high and low traffic patterns are considered to highlight the capability of the proposed CRBO algorithm. In high traffic scenario, almost $90 \%$ of the nodes are involved in active communication at any instant. But in low traffic, only $20 \%$ of the nodes in the network are participating in the communication. As expected, the contention and congestion level in high traffic scenario is more than in low traffic scenario. In these two extreme cases, the proposed CRBO algorithm outperforms BEB and MADCF as depicted in the following sections. The other simulation parameters are listed in Table 2.

Table 2. Simulation Parameters

\begin{tabular}{|c|c|}
\hline Parameters & Values \\
\hline Area & 1000 X 1000 \\
\hline Physical Layer \& Antenna & DSSS \& Omni directional \\
\hline MAC protocol & 802.11 DCF \\
\hline Routing Protocol & AODV \\
\hline Traffic type \& Packet size & CBR \& 512 bytes \\
\hline Topology & Random \& Grid topology \\
\hline DIFS \& SIFS & $50 \mathrm{~s} \mathrm{\&} 10 \mathrm{~s}$ \\
\hline Slot time & $20 \mathrm{~s}$ \\
\hline Transmission Range & $250 \mathrm{~m}$ \\
\hline Simulation time & $180 \mathrm{sec}$ \\
\hline
\end{tabular}

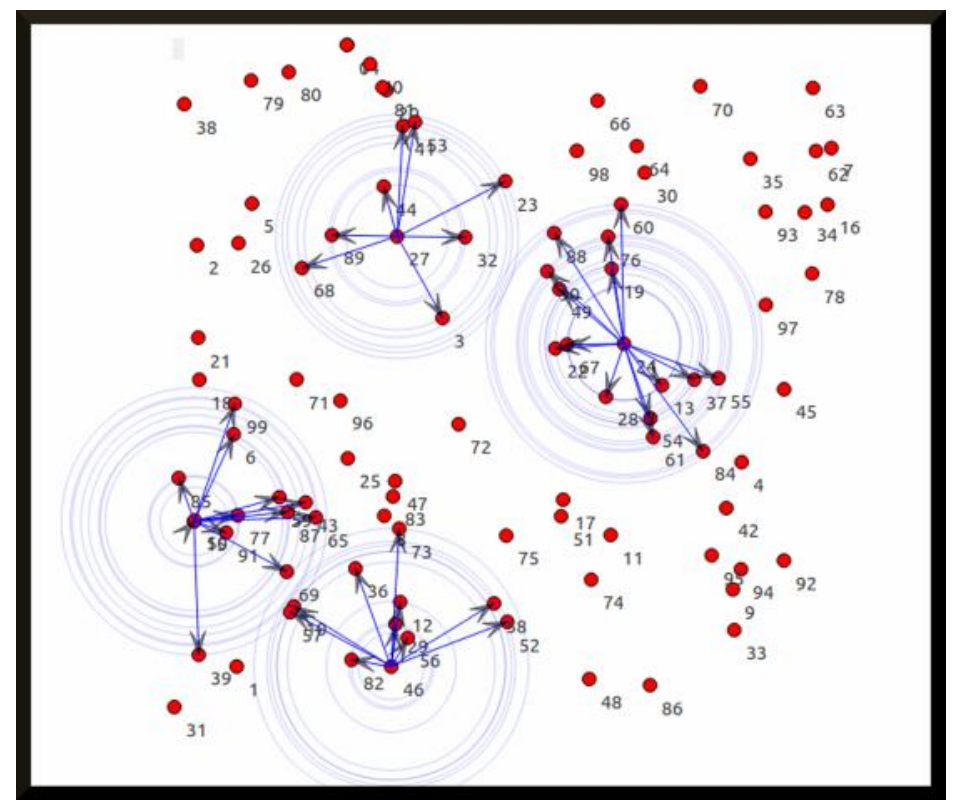

Figure 2. Random Topology with 100 nodes 


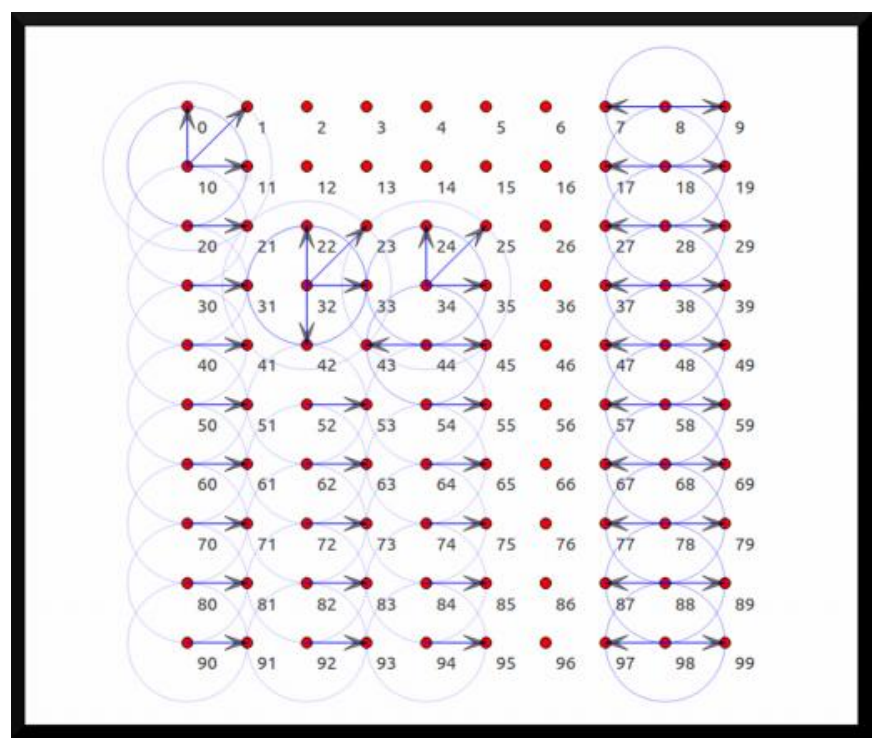

Figure 3. Grid Topology with 100 nodes

4.1 Throughput performance: Figures 4 and 5 show the throughput performance of BEB, MADCF and CRBO in random topology with varying number of nodes under high and low traffic scenario respectively. The throughput is also measured for these three algorithms in grid topology under two different traffic scenarios and which are given in Figures 6 and 7 respectively. It is inferred from these Figures that, the throughput performance depends on contention window size at different backoff stage. Irrespective of the topology and traffic type, the proposed CRBO algorithm performs well as compared the legacy BEB algorithm and MADCF. This is mainly due to the dynamic adaptiveness of the proposed algorithm to estimate $\mathrm{CW}$ size by considering the present status of the network. Collision ratio, one of the decision parameters in CRBO algorithm, reveals the degree of the congestion in the network. With the aid of this, the proposed algorithm updates CW size linearly in case of low congestion or exponentially in high congestion in the network. As a consequence, the waiting time to capture the idle medium is appropriately estimated to successfully transmit more number of packets or waiting for the idle medium without disturbing ongoing transmissions. This leads to high throughput by minimizing number of collisions among nodes. In addition to this, the proposed algorithm predicts $\mathrm{CW}$ size after having successful transmission. Unlike the resetting mechanism in BEB, the proposed CRBO utilizes the decision parameter, retransmission count, to determine the $\mathrm{CW}$ size. Due to this dynamic updation, the anomalies of resetting mechanism as mentioned in Section 3.2 are minimized thereby enhancing throughput performance in both the topologies.

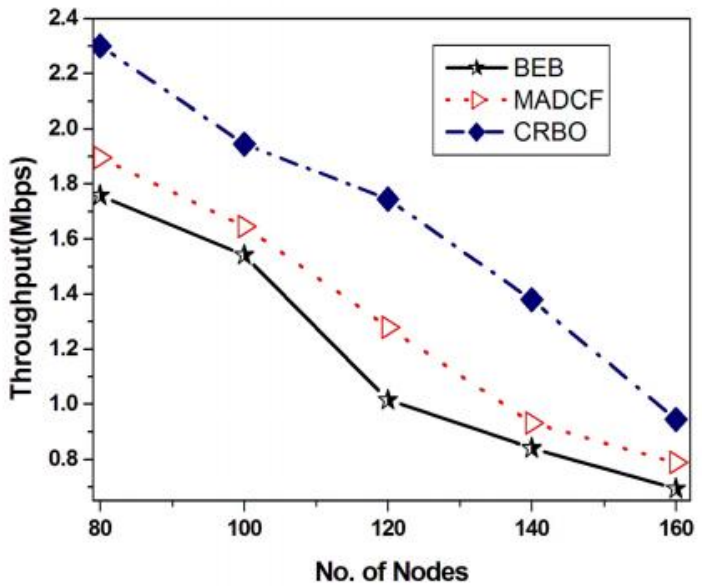

Figure 4. Throughput in Random topology under High Traffic

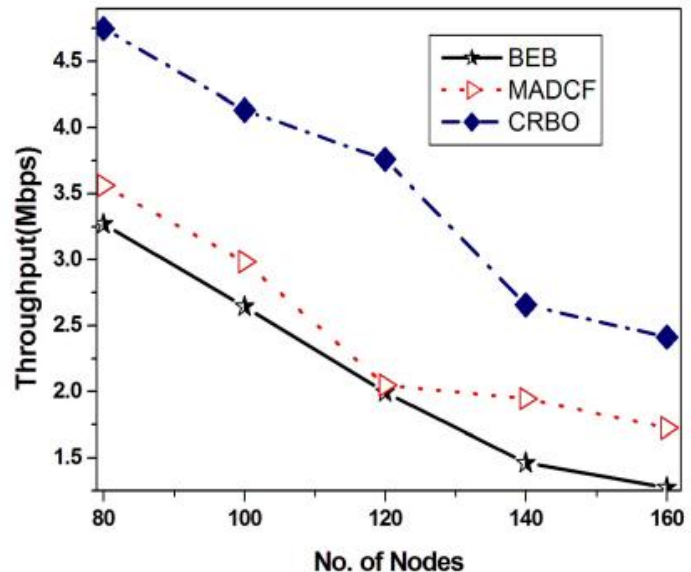

Figure 5.Throughput in Random topology under Low Traffic 


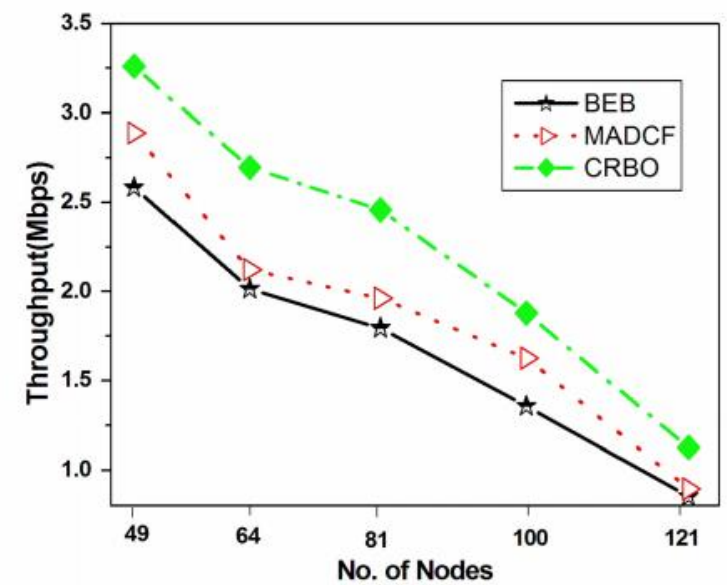

Figure 6. Throughput in Grid topology under High Traffic

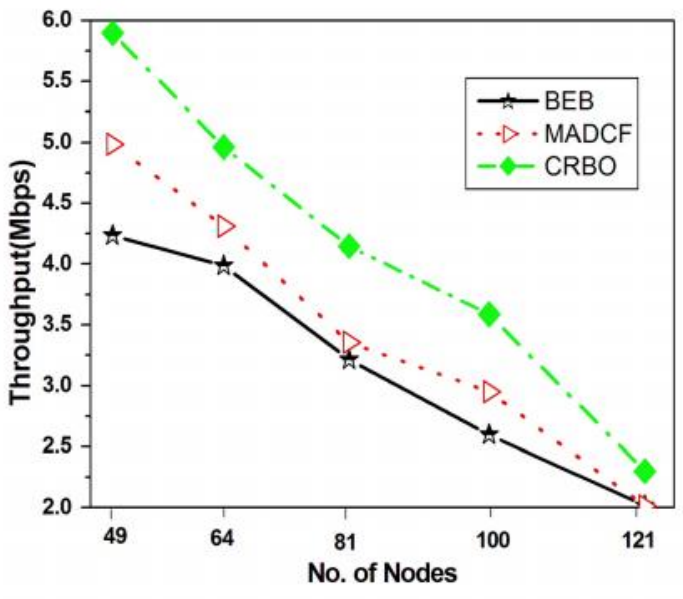

Figure 7.Throughput in Grid topology under Low Traffic

4.2 Delay performance: The end-to-end delay performance for each algorithm is measured in random topology and shown in Figures 8 and 9 under high and low traffic conditions respectively. Also in grid topology, delay is estimated under high and low traffic and shown in Figures 10 and 11 respectively. From these figures, it is deduced that BEB and MADCF algorithms have experienced high end-to-end delay as they exhibited long idle time after collisions (specifically at the end of backoff stages) which consequently increases delay. Whereas in the proposed CRBO algorithm, $\mathrm{CW}$ is updated either exponentially or linearly to reduce the unnecessary idle time as much as possible. Moreover, the CW adaptation after the successful transmission in the proposed algorithm helps to minimize the collision probability as it forces the nodes to wait before attempting the next unsafe transmissions. As a result the time taken for a packet to travel from one station to another station decreases thereby minimizing end -to-end delay under different traffic patterns.

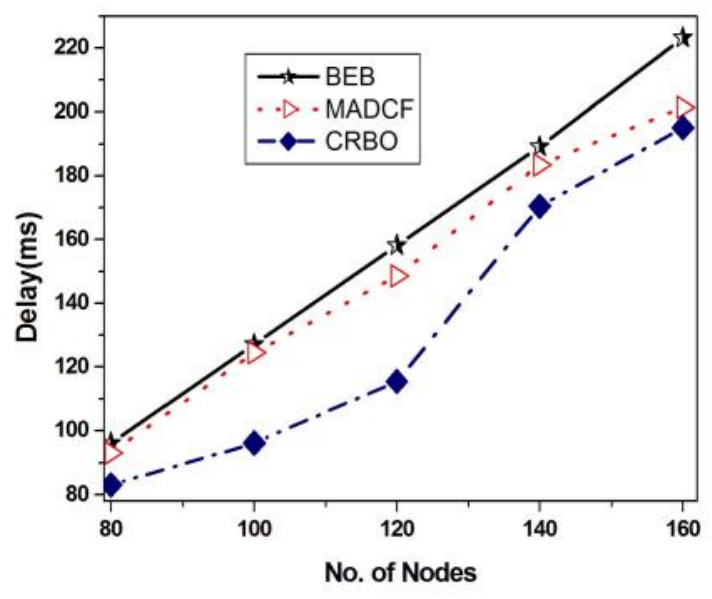

Figure 8. Delay in Random topology under High Traffic

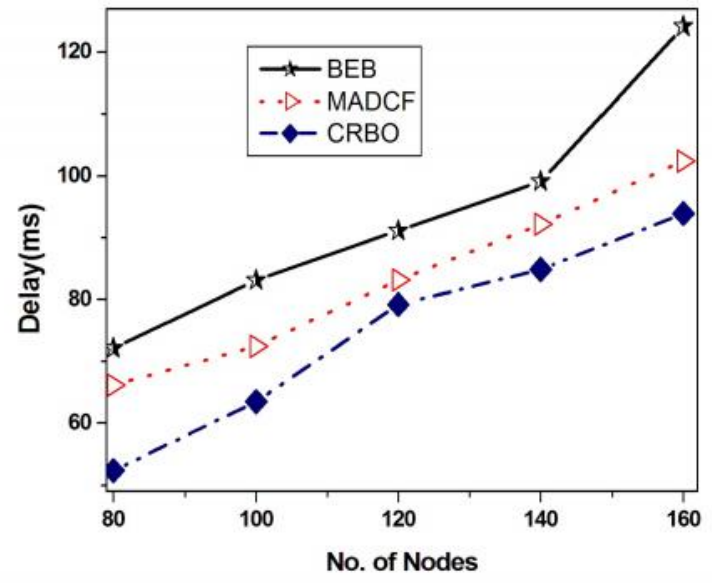

Figure 9.Delay in Random topology under Low Traffic 


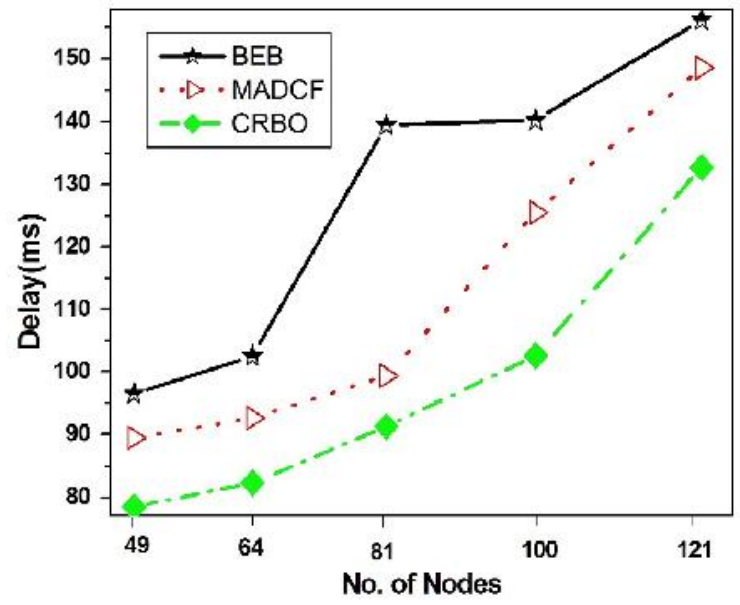

Figure 10. Delay in Grid topology under High Traffic

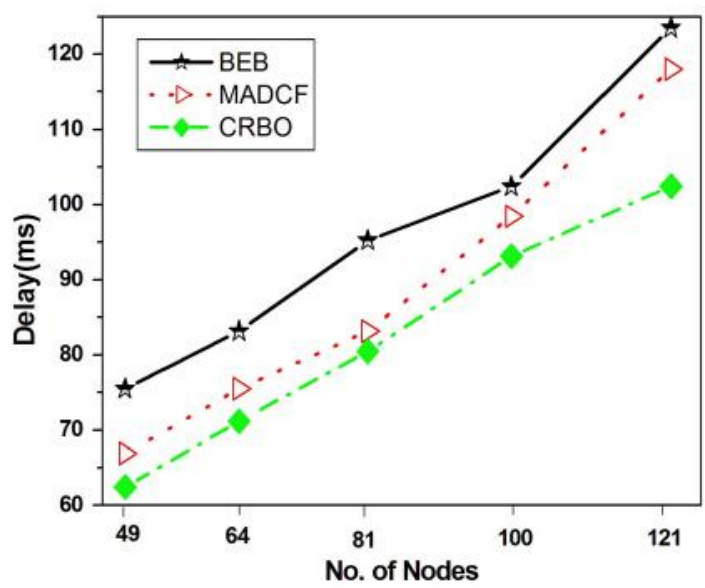

Figure 11.Delay in Grid topology under Low Traffic

4.3 Packet loss performance: The packet loss parameter is calculated as the ratio of the number of packets dropped to the number of total packets sent. The collision rate is related to the packet loss ratio since the number of packets dropped are an indirect measure of the number of collisions in the system. Figures 12 and 13 show the packet loss incurred by BEB, MADCF and CRBO algorithms in random topology under heavy and low traffic respectively. As depicted in these figures, packet loss is increased as the number of active contending stations are increased. Figures 14 and 15 reveal the packet loss performance of these algorithms in grid topology under high and low traffic respectively. It is inferred from these figures that the packet loss ratio is more in random topology as it incorporates node mobility than in grid topology. However, the proposed CRBO algorithm performs better than the legacy $\mathrm{BEB}$ and MADCF. In the proposed $\mathrm{CRBO}$ algorithm, each node is assigned the appropriate $\mathrm{CW}$ size according to collision ratio and retransmission count. So the probability of selecting the same contention window size by more than one node is minimized thereby ensuring different waiting time assigned to the stations. This guarantees either the successful transmission or defer from accessing the busy medium to minimize collisions as legacy BEB and MADCF.

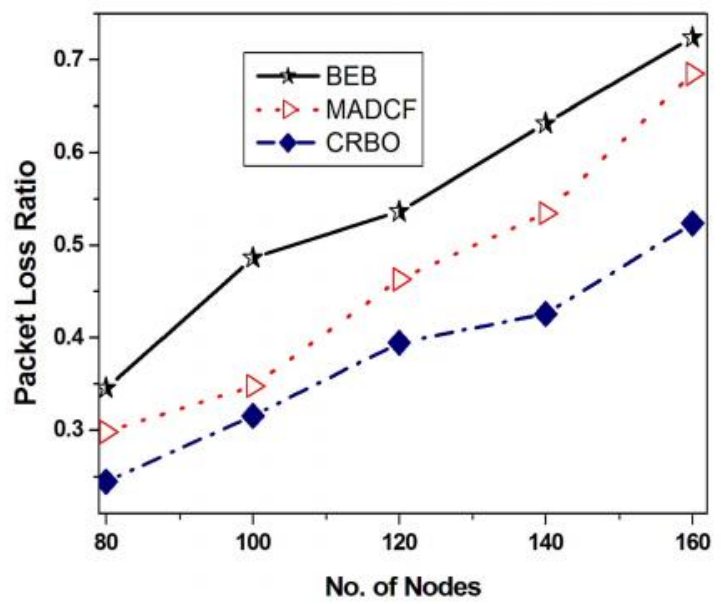

Figure 12. Packet loss in Random topology under High Traffic

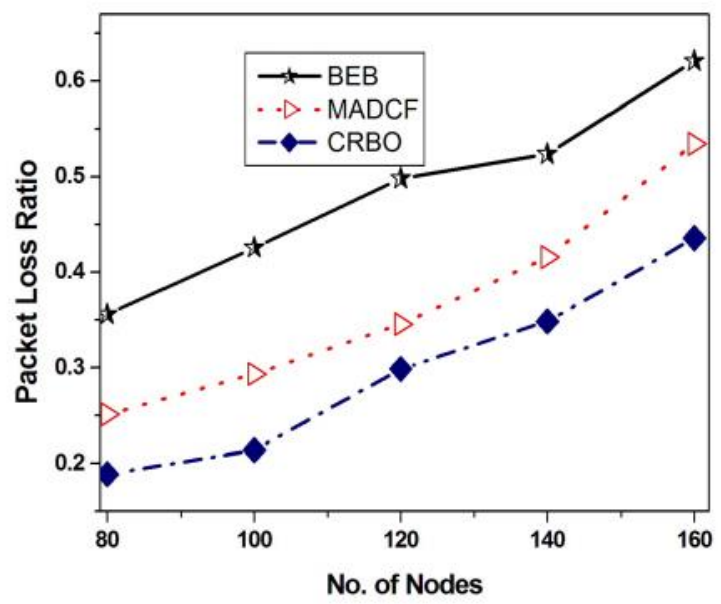

Figure 13. Packet loss in Random topology under Low Traffic 


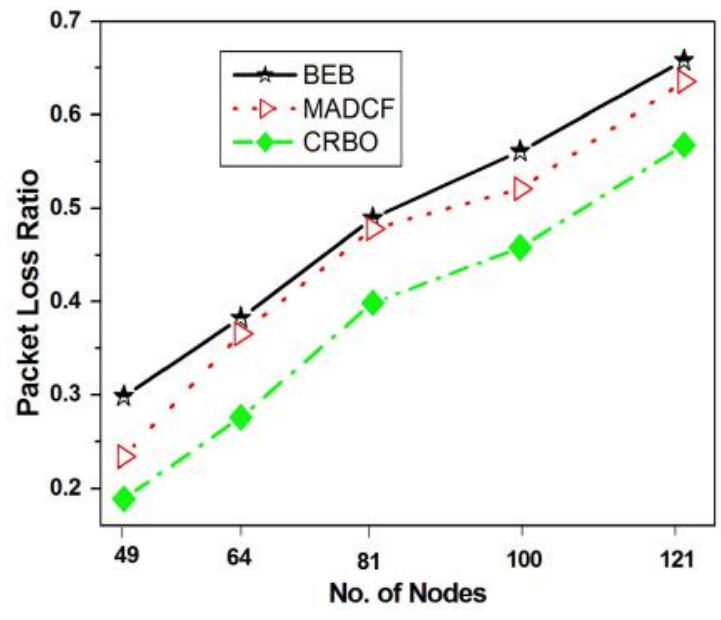

Figure 14. Packet loss in Grid topology under High Traffic

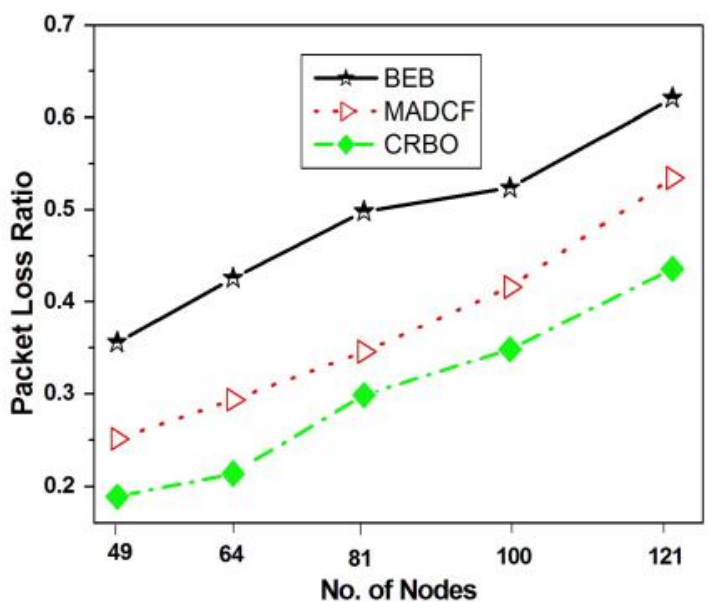

Figure 14. Packet loss in Grid topology under Low Traffic

4.4 Fairness Index performance: The fairness index measure is used to determine whether the stations are receiving a fair share of the system resources. The Jain's fairness index equation in (5) (Jain et al,2016) is given by,

$$
\mathrm{J}\left(\mathrm{x}_{1}, \mathrm{x}_{2}, \ldots, \mathrm{x}_{\mathrm{n}}\right)=\left(\sum_{i=1}^{n} x_{i}\right)^{2} / n *\left(\sum_{i=1}^{n} x_{i}^{2}\right)
$$

Using the above equation, the fairness index attained by BEB, MADCF and the proposed CRBO algorithms are measured in random and grid topology under high and low traffic scenarios. These are given in Figures 15, 16, 17 and 18. From these figures, it can be stated that the proposed CRBO algorithm achieves better fairness index than the legacy BEB and MADCF algorithm. One of the main reasons is that adjusting $\mathrm{CW}$ size after having successful transmission instead of resetting $\mathrm{CW}$ size to its minimum value. This dynamic adjustment of $\mathrm{CW}$ is stimulated by collision rate and retransmission count. As a consequence, stations that had successful transmission will not preempt the longer waiting station thereby leading to fair allocation of medium among competing nodes.

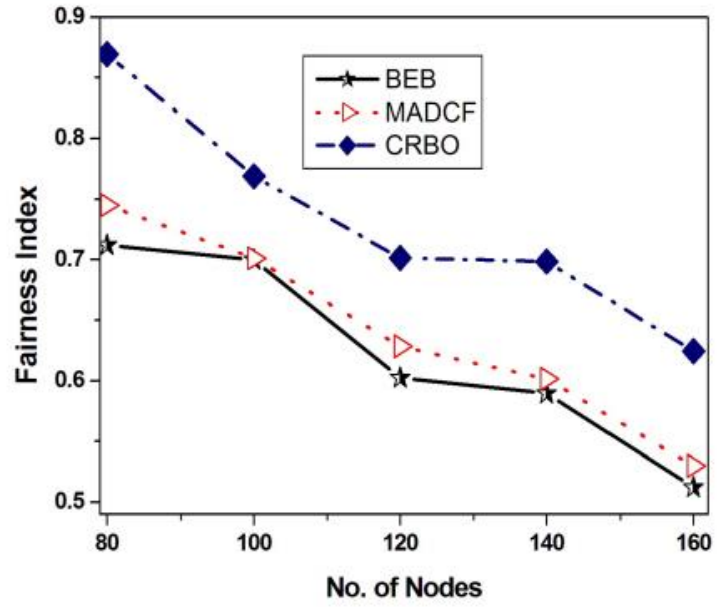

Figure 15. Fairness index in Random topology under High Traffic

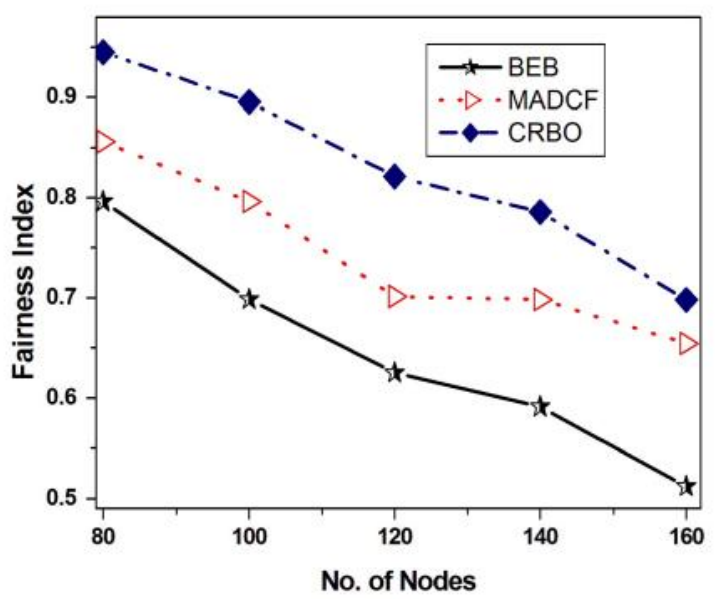

Figure 16. Fairness index in Random topology under Low Traffic 


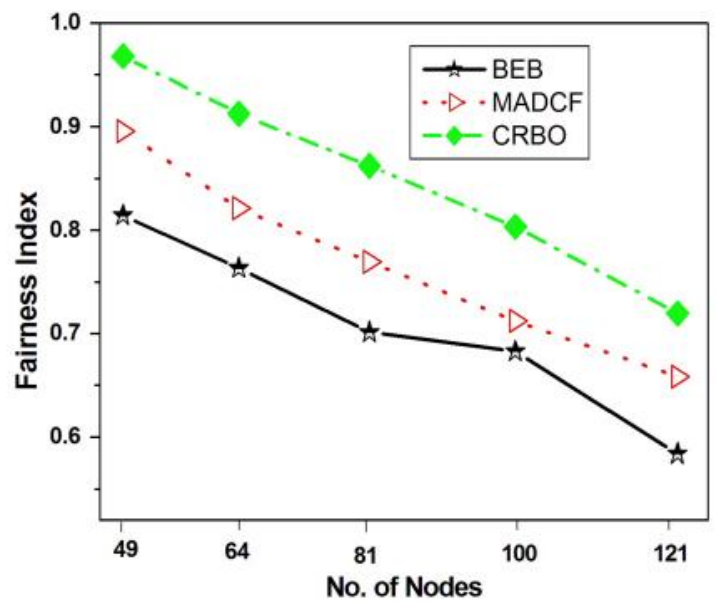

Figure 17. Fairness index in Grid topology under High Traffic

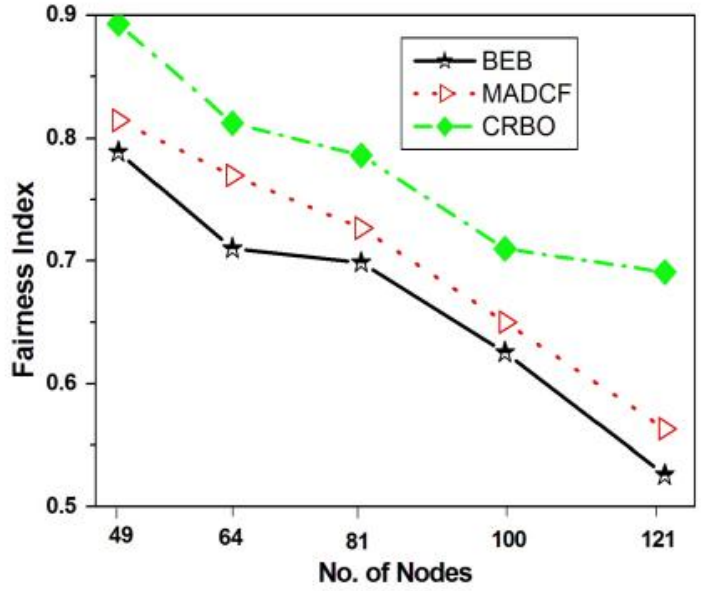

Figure 18.Fairness index in Grid topology under Low Traffic

\section{Conclusion}

This paper has proposed CRBO algorithm to enhance the network performance under heavy and low traffic in the random and grid network topology. In the proposed algorithm, $\mathrm{CW}$ is incremented either exponentially or linearly based on collision rate. In case of successful transmission, the resetting or polynomial $\mathrm{CW}$ adjustment based on retransmission count is adopted. These dynamic adaptations of $\mathrm{CW}$ in the proposed algorithm guarantee more efficient resource utilization by minimizing delay and packet loss. The possible extension of the proposed algorithm is to use cross layer information to better distinguish the wireless environment and according to that CW size may be changed. From the physical layer, Signal to Interference Noise Ratio (SINR) information can be utilized to estimate the channel quality. This piece of information can be utilized by the proposed algorithm for better scheduling of transmissions with minimum of overhead.

\section{References}

Alkadeki H.,Wang X.,Odetayo M. 2016.Improving Performance of IEEE 802.11 by a Dynamic Control Backoff Algorithm Under Unsaturated Traffic Loads. arXiv preprint arXiv:1601.00122.

Bharghavan.V., Demers.A.,Shenker.S.,Zhang.L.1994. MACAW: a media access protocol for wireless LAN's. ACM SIGCOMM

Computer Communication Review, Vol. 24, No. 4,pp. 212-225.

Brenner P. 1997.A technical tutorial on the IEEE 802.11 protocol. BreezeCom Wireless Communication, Vol. 1.

Cho Y.C., Yoon S.J., Ko Y.B. 2011.Modifying the IEEE 802.11 MAC protocol for multi-hop reservation in MIMC tactical ad hoc networks. IEEE Workshops of International Conference on Advanced Information Networking and Applications (WAINA), pp. 178-183.

Deng.J.,Varshney P.K.,Haas Z.J.2004. A new backoff algorithm for the IEEE 802.11 distributed coordination function. Proceedings of CNDS, pp.18-21.

Elarbaoui.I.,Refai H.H.2008. Enhancement of IEEE 802.11 DCF backoff algorithm under heavy traffic. Computer Systems and Applications, IEEE/ACS International Conference, AICCSA 2008, pp. 1082-1087.

Huang Y.,Wang Y.,Zhu R.,Chen X.,Meng Q. 2016. Synchronized contention windows-based backoff algorithm in IEEE 802.11 wireless networks. IEEE International Conference on Computer, Information and Telecommunication Systems (CITS), pp. 1-5.

Jain R., Chiu D.M., Hawe W.R.1984. A quantitative measure of fairness and discrimination for resource allocation in shared computer system. Eastern Research Laboratory, Digital Equipment Corporation Hudson, MA, Vol. 38.

Jochen H. Schiller.2003.Mobile Communication. Second Edition, Pearson Education.

Natkaniec M., Kosek-Szott K., Szott S., Bianchi G. 2013.A survey of medium access mechanisms for providing QoS in ad-hoc networks. IEEE communications surveys \& tutorials. Vol.15, No. 2, pp. 592-620.

Thorpe C., Murphy L. 2014.A survey of adaptive carrier sensing mechanisms for IEEE 802.11 wireless networks. IEEE Communications Surveys \& Tutorials. Vol.16, No.3,pp. 1266-1293.

Teerawat I., Ekram H. 2009.Introduction to Network Simulator NS2. Springer Publication. 
Zhang.C., Chen. P., Ren. J., Wang. X., Vasilakos A.V. 2016.A backoff algorithm based on self-adaptive contention window update factor for IEEE 802.11 DCF. Wireless Networks, pp. 1-10.

Zhai H., Wang J., Chen X., Fang Y.2006. Medium access control in mobile ad hoc networks: challenges and solutions. Wireless Communications and Mobile Computing. Vol. 6, No. 2, pp.151-70.

Bononi L., Marco C., Enrico G. 2004. Runtime optimization of IEEE 802.11 wireless LANs performance.IEEE Transactions on Parallel and Distributed Systems. Vol. 15, No.1, pp.66-80.

Hong K., Lee S., Kim K., Kim Y. 2012. Channel condition based contention window adaptation in IEEE 802.11 WLANs. IEEE Transactions on Communications, Vol. 60, No. 2, pp. 469-478.

\section{Biographical notes}

B. Nithya received Ph.D. degree from National Institute of Technology, Trichy in 2015.She is working as an Assistant Professor in National Institute of technology (NIT) Trichy since 2007.Her research interests include wireless networks, Mobile Computing and Optimization Techniques.

A. Justin Gopinath is currently pursuing Ph.D. degree in Computer Science and Engineering, National Institute of Technology, Trichy, India. He received B.E and M.Tech degree in 2004 and 2010 respectively. He has 12 years of experience in teaching. His area of research includes Internet of Things (IoT), Wireless Networks.

Venkatesh Kameswaran is currently pursuing B.Tech degree in Computer Science and Engineering, National Institute of Technology, Trichy,India.

P. Yogesh is currently pursuing B.Tech. degree in Computer Science and Engineering, National Institute of Technology, Trichy,India.

Received April 2017

Accepted April 2017

Final acceptance in revised form May 2017 